\title{
A Simplified Version of the Fast Algorithms of Brent and Salamin
}

\author{
By D. J. Newman*
}

\begin{abstract}
We produce more elementary algorithms than those of Brent and Salamin for, respectively, evaluating $e^{x}$ and $\pi$. Although the Gauss arithmetic-geometric process still plays a central role, the elliptic function theory is now unnecessary.
\end{abstract}

In their remarkable papers, Brent [1] and Salamin [3], respectively, used the theory of elliptic functions to obtain "fast" computations of the function $e^{x}$ and of the number $\pi$. In both cases rather heavy use of elliptic function theory, such as the transformation law of Landen, had to be utilized. Our purpose, in this note, is to give a highly simplified version of their constructions. In our approach, for example, the incomplete elliptic integral is never used.

We begin as they did with the Gauss arithmetic-geometric process, $T(a, b)=$ $((a+b) / 2, \sqrt{a b})$ which maps couples with $a \geqslant b>0$ into same. From the inequalities

$$
\frac{(a+b) / 2-\sqrt{a b}}{(a+b) / 2+\sqrt{a b}}=\left(\frac{\sqrt{a}-\sqrt{b}}{\sqrt{a}+\sqrt{b}}\right)^{2} \leqslant\left(\frac{a-b}{a+b}\right)^{2},
$$

and

$$
\frac{(a+b) / 2}{\sqrt{a b}} \leqslant \frac{a}{\sqrt{a b}}=\sqrt{\frac{a}{b}},
$$

we see that $T^{i}(a, b)$ goes to its limiting couple $(m, m)(m=m(a, b)$ the so-called arithmetic-geometric mean) with "quadratic" speed. Indeed, $m(a, b)$ is determined to $n$ places for an $i$ of around $\log \log a / b+\log n$. The $\log \log$ from the $\sqrt{a / b}$ inequality expressing the time till the ratio first goes below 2 , and the log from the $((a-b) /(a+b))^{2}$ inequality expressing the time for the error squaring to do its job.

Next, we recall Gauss' beautiful formula:

$$
m(a, b)=\pi / \int_{-\infty}^{\infty} \frac{d x}{\sqrt{\left(x^{2}+a^{2}\right)\left(x^{2}+b^{2}\right)}}
$$

Received October 7, 1981; revised November 17, 1981, and March 22, 1984.

1980 Mathematics Subject Classification. Primary 65D15, 33A25, 41A25.

*Supported in part by NSF Grant MGS 7802171. 
which follows from the fact that this (complete) elliptic integral is invariant under $T$. This fact, that namely

$$
\int_{-\infty}^{\infty} \frac{d x}{\sqrt{\left(x^{2}+a^{2}\right)\left(x^{2}+b^{2}\right)}}=\int_{-\infty}^{\infty} \frac{d t}{\sqrt{\left(t^{2}+((a+b) / 2)^{2}\right)\left(t^{2}+a b\right)}},
$$

is a simple consequence of the change of variables $t=(x-a b / x) / 2$. Namely, we obtain

$$
\begin{gathered}
d t=\frac{x^{2}+a b}{2 x^{2}} d x, \quad t^{2}+\left(\frac{a+b}{2}\right)^{2}=\frac{\left(x^{2}+a^{2}\right)\left(x^{2}+b^{2}\right)}{4 x^{2}}, \\
t^{2}+a b=\frac{\left(x^{2}+a b\right)^{2}}{4 x^{2}},
\end{gathered}
$$

$0<x<\infty$, so that indeed we have

$$
\begin{aligned}
\int_{-\infty}^{\infty} \frac{d x}{\sqrt{\left(x^{2}+a^{2}\right)\left(x^{2}+b^{2}\right)}} & =\int_{0}^{\infty} \frac{2 d x}{\sqrt{\left(x^{2}+a^{2}\right)\left(x^{2}+b^{2}\right)}} \\
& =\int_{-\infty}^{\infty} \frac{d t}{\sqrt{\left(t^{2}+((a+b) / 2)^{2}\right)\left(t^{2}+a b\right)}}
\end{aligned}
$$

Accordingly, a repeated use of this invariance gives

$$
\begin{aligned}
\int_{-\infty}^{\infty} \frac{d x}{\sqrt{\left(x^{2}+a^{2}\right)\left(x^{2}+b^{2}\right)}} & =\cdots=\int_{-\infty}^{\infty} \frac{d x}{\sqrt{\left(x^{2}+m^{2}\right)\left(x^{2}+m^{2}\right)}} \\
& =\int_{-\infty}^{\infty} \frac{d x}{x^{2}+m^{2}}=\frac{\pi}{m},
\end{aligned}
$$

and this is exactly Gauss' formula.

Actually, it is handier for us to work with what we might call the harmonic-geometric mean which can be defined by $h(a, b)=a b / m(a, b)$ or, alternatively, as the limit under repeated applications of $S$, rather than $T$, where

$$
S(a, b)=(\sqrt{a b}, 2 a b /(a+b)) .
$$

In these terms Gauss' formula reads

$$
h(a, b)=\frac{1}{\pi} \int_{-\infty}^{\infty} \frac{d x}{\sqrt{\left(1+x^{2} / a^{2}\right)\left(1+x^{2} / b^{2}\right)}} .
$$

The only place that we actually use this formula is to establish the asymptotic formula:

$$
h(N, 1)=\frac{2}{\pi} \log 4 N+O\left(1 / N^{2}\right) .
$$

(This simple-looking formula certainly deserves an elementary proof independent of elliptic integrals, but we are unable to find one.)

So begin with

$$
h(N, 1)=\frac{2}{\pi} \int_{0}^{\infty} \frac{d x}{\sqrt{\left(1+x^{2}\right)\left(1+x^{2} / N^{2}\right)}}
$$


and observe that the map $x \rightarrow N / x$ leaves the integrand invariant. Thereby, we conclude

$$
\int_{0}^{\sqrt{N}} \frac{d x}{\sqrt{\left(1+x^{2}\right)\left(1+x^{2} / N^{2}\right)}}=\int_{\sqrt{N}}^{\infty} \frac{d x}{\sqrt{\left(1+x^{2}\right)\left(1+x^{2} / N^{2}\right)}}
$$

which gives us

$$
\begin{aligned}
h(N, 1) & =\frac{4}{\pi} \int_{0}^{\sqrt{N}} \frac{d x}{\sqrt{\left(1+x^{2}\right)\left(1+x^{2} / N^{2}\right)}} \\
& =\frac{4}{\pi} \int_{0}^{\sqrt{N}} \frac{1}{\sqrt{\left(1+x^{2}\right)}}\left(1-x^{2} / 2 N^{2}+O\left(x^{4} / N^{4}\right)\right) d x \\
& =\frac{4}{\pi} \int_{0}^{\sqrt{N}}\left(\frac{1}{\sqrt{1+x^{2}}}-\frac{x}{2 N^{2}}\right) d x+O\left(1 / N^{2}\right) \\
& =\frac{4}{\pi}(\log (\sqrt{N}+\sqrt{N+1})-1 / 4 N)+O\left(1 / N^{2}\right)
\end{aligned}
$$

and so, since

$$
\sqrt{N}+\sqrt{N+1}=2 \sqrt{N}(1+1 /(2 N+2 \sqrt{N(N+1)}))
$$

we obtain

$$
\log (\sqrt{N}+\sqrt{N+1})=\log 2 \sqrt{N}+1 / 4 N+O\left(\frac{1}{N^{2}}\right)
$$

which together with the previous gives

$$
h(N, 1)=\frac{4}{\pi} \log 2 \sqrt{N}+O\left(\frac{1}{N^{2}}\right)=\frac{2}{\pi} \log 4 N+O\left(\frac{1}{N^{2}}\right),
$$

as required. (This result can also be found in [2].)

Summarizing, then, we have produced a fast method for obtaining $n$ places of $2 \log 4 N / \pi$ (if $N$ is of the size $c^{n}$ ). But, and here is the trick, this combination of $\pi$ and the logarithm can be used to yield both of them separately, and we can thereby rederive both Salamin's and Brent's results.

To obtain $\pi$ we examine the difference, $h(N+1,1)-h(N, 1)$, and observe that $N(h(N+1,1)-h(N, 1))=2 / \pi+O(1 / N)$ which gives $n$ place accuracy for $\pi$ if we choose, e.g., $N=2^{n}$.

For the logarithm, on the other hand, we look to the quotient, $h(N+1,1) / h(N, 1)$. This time we obtain

$$
N\left(\frac{h(N+1,1)}{h(N, 1)}-1\right)=N \frac{\log (1+1 / N)+O\left(1 / N^{2}\right)}{\log 4 N+O(1 / N)}=\frac{1}{\log 4 N+O(1 / N)} .
$$

From this we will be able to evaluate $\log x$ throughout the interval $(3,9)$, and so, of course, throughout any interval. And thereby, we will be able to obtain $e^{x}$, the inverse function, by the usual use of the (fast) Newton iteration scheme.

To obtain $\log x$, then, in the interval $(3,9)$, we first calculate $N=\frac{1}{4} x^{n}$, a process that takes only $\log n$ multiplications. But then the above formula becomes, upon substitution of this value of $N$,

$$
\frac{1}{4} n x^{n}\left(\frac{h\left(\frac{1}{4} x^{n}+1,1\right)}{h\left(\frac{1}{4} x^{n}, 1\right)}-1\right)=\frac{1}{\log x}+O\left(\frac{n}{x^{n}}\right)=\frac{1}{\log x}+O\left(\frac{n}{3^{n}}\right)
$$

which does give the desired $n$ place evaluation of $\log x$. 
This trick of "differencing" $h(N+1,1)$ and $h(N, 1)$, of course, carries a price. Thus we must compute these two quantities to $2 n$ places and so the running time is around twice as long as the corresponding ones of Brent and Salamin.

Mathematics Department

Temple University

Broad and Montgomery Ave.

Philadelphia, Pennsylvania 19122

1. R. Brent, "Fast multiple evaluation of elementary functions," J. Assoc. Comput. Mach., v. 23, 1976, pp. 242-251.

2. R. BRENT, Multiple-Precision Zero-Finding Methods and the Complexity of Elementary Function Evaluation (J. F. Traub, ed.), Academic Press, New York, 1975, pp. 151-176.

3. E. Salamin, “Computation of $\pi$ using the arithmetic-geometric mean," Math. Comp., v. 30, 1976, pp. $565-570$. 